\title{
Bayesian Inference: The Enabler of Mindsponge Philosophy
}

\author{
Minh-Hoang Nguyen \\ Centre for Interdisciplinary Social Research \\ Phenikaa University
}

Yen Nghia, Ha Dong, Hanoi 100803, Vietnam

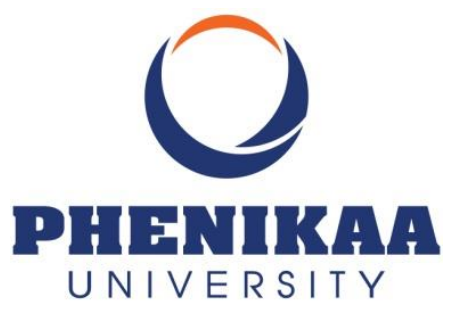

February 15, 2021

A human's brain is a very complex system consisting of approximately 86 billion neurons. Such enormous nervous system influences and is influenced by other body systems of the human [1].

For better understanding the human's mind, researchers in cognitive and psychological sciences are, therefore, required to obtain a model or theory that is adequate to scope its dynamic and multiplex mechanism.

In a previous essay, I proposed the Mindsponge mechanism as a potential model for examining one of the most extreme thoughts and behaviors of an individual - suicidal ideation and behaviors, due to its dynamics and multiplexity [2]. However, what kind of method can be employed given such dynamics and multiplexity?

My colleagues and I also suggest that Bayesian inference, specifically the bayesvl $\mathrm{R}$ package, can work together quite well [3-6]. In this essay, I would like to explain how the Bayesian and Mindsponge notions are a good fit for each other.

Regarding the Mindsponge mechanism [7,8], three prominent characteristics can be listed as follows:

1. The Mindsponge process is influenced by both external information and internal preferences (or the mindset's cost-benefit judgment). 
2. The causal nexus within the Mindsponge is non-linear rather than linear due to the multiplex nature of information processing.

3. The Mindsponge process is updating in nature through inductive reasoning.

Because of these characteristics, not many statistics approaches are capable of investigating Mindsponge philosophy. Among the applicable methods, Bayesian inference stands out as an enabler of the philosophy. Firstly, the Bayesian computation aided by the Markov Chain Monte Carlo (MCMC) algorithms offers researchers extreme flexibility in constructing the model.

Specifically, the MCMC algorithms iteratively generate a large number of samples that help estimate the posterior distribution of any parameters with high precision $[9,10]$.

This advantage enables a wide range of models that can be fitted, such as multilevel models, non-linear model frameworks, or a mixture of both. Therefore, it allows researchers to take the first and second characteristics of Mindsponge philosophy into consideration.

Secondly, Bayesian inference treats all properties as probability [11], which permits researchers to look solely at certain aspects of a whole picture without controlling other parameters. Given the dynamics, multiplexity, and updating manner of the Mindsponge process, the number of control parameters needed for reducing the model's residual might be an enormous number that substantially increases the research cost and eventually reduces the research feasibility [12].

Moreover, the higher number of control variables are added, the easier scientific misconducts can be actualized. For example, inappropriate practices, such as stargazing, p-hacking, and HARKing, can be conducted by capitalizing the sample-to-sample variability in the $\mathrm{p}$-value [13-15]. Hence, besides assisting researchers in framing research matters, Bayesian inference can also help reduce the persistent reproducibility crisis in psychological research [16,17].

Thirdly, the Bayesian belief updating process plays a very fundamental role in using the Mindsponge philosophy. Humans live in a world with an excessive amount of information being updated daily, or even hourly, so their psychological traits and behaviors can change rapidly depending on the context and personal demand (or mindset's preferences).

As a result, there are possibilities that published scientific findings can be outdated and cannot be replicated after some time. With the belief updating function, Bayesian inference is, thus, a well-fitted methodology for studying cognitive and psychological matters using the Mindsponge philosophy. 
To conclude, Bayesian inference aided by the MCMC technique and Mindsponge mechanism fits each other closely in studying cognitive and psychological issues. Further research employing the Mindsponge mechanism is encouraged to consider the bayesvl package as a potential alternative since they have been developed by the same person using an almost similar philosophy. Our team's recent publication is a typical exemplary demonstration of how Bayesian inference and Mindsponge mechanism could be meticulously combined [18].

\section{References}

[1] Suzana Herculano-Houzel. (2012). The remarkable, yet not extraordinary, human brain as a scaled-up primate brain and its associated cost. Proceedings of the National Academy of Sciences, 109, 10661-10668.

[2] Minh-Hoang Nguyen. (2021). Suicidal Ideation and Behavior Rethinking: The Mindsponge Approach. OSF Preprints. DOI: 10.31219/osf.io/ec67s.

[3] Minh-Hoang Nguyen et al. (2021). On the working of the mindsponge concept and the bayesvl R package. OSF Preprints. DOI: 10.31219/osf.io/jhg7b.

[4] Quan-Hoang Vuong et al. (2020). Bayesian analysis for social data: A step-by-step protocol and interpretation. MethodsX, 7, 100924.

[5] Quan-Hoang Vuong et al. (2020). Improving Bayesian statistics understanding in the age of Big Data with the bayesvl R package. MethodsX, 4, 100016.

[6] Viet-Phuong La \& Quan-Hoang Vuong. (2019). bayesvl: Visually learning the graphical structure of Bayesian networks and performing MCMC with 'Stan'. The Comprehensive $R$ Archive Network (CRAN). Available from: https://cran.rproject.org/web/packages/bayesvl/index.html.

[7] Quan-Hoang Vuong. (2016). Global mindset as the integration of emerging sociocultural values through mindsponge processes: A transition economy perspective. In J. Kuada (Ed.), Global Mindsets: Exploration and Perspectives (pp. 109-126). London: Routledge.

[8] Quan-Hoang Vuong \& Nancy K. Napier. (2015). Acculturation and global mindsponge: an emerging market perspective. International Journal of Intercultural Relations, $49,354-367$.

[9] Don van Ravenzwaaij, Pete Cassey, \& Scott D. Brown. (2016). A simple introduction to Markov Chain Monte-Carlo sampling. Psychonomic Bulletin Review, 25(1), 143-154. 
[10] David B. Dunson. (2001). Commentary: practical advantages of Bayesian analysis of epidemiologic data. American Journal of Epidemiology, 153(12), 1222-1226.

[11] Peter J. Green et al. (2015). Bayesian computation: a summary of the current state, and samples backwards and forwards. Statistics Computing, 25(4), 835-862.

[12] Quan-Hoang Vuong. (2018). The (ir)rational consideration of the cost of science in transition economies. Nature Human Behaviour, 2(1), 5-5.

[13] Lewis G Halsey et al. (2015). The fickle P value generates irreproducible results. Nature Methods, 12(3), 179-185.

[14] Quan-Hoang Vuong, Manh-Tung Ho, \& Viet-Phuong La. (2019). 'Stargazing' and phacking behaviours in social sciences: some insights from a developing country. European Science Editing, 45(2), 54-55.

[15] Norbert L. Kerr. (1998). HARKing: Hypothesizing after the results are known. Personality Social Psychology Review, 2(3), 196-217.

[16] Monya Baker. (2016). 1,500 scientists lift the lid on reproducibility. Nature News, 533(7604), 452-454. Available from: https://www.nature.com/news/1-500scientists-lift-the-lid-on-reproducibility-1.19970

[17] Open Science Collaboration. (2015). Estimating the reproducibility of psychological science. Science, 349(6251), aac4716.

[18] Minh-Hoang Nguyen et al. (2021). Alice in Suicideland: Exploring the Suicidal Ideation Mechanism through the Sense of Connectedness and Help-Seeking Behaviors. International Journal of Environmental Research and Public Health, 18(7), 3681 . 